Publication List

Juntendo Medical Journal

2018. 64 (3), 235-248

\title{
Publications from Juntendo University Graduate School of Medicine, 2016 [1/6]
}

\section{Department of Microbiology}

〈Orginal Articles〉

1) Katayama $Y$, Sekine M, Hishinuma T, Aiba $Y$, Hiramatsu K: Complete Reconstitution of the Vancomycin-Intermediate Staphylococcus aureus Phenotype of Strain Mu50 in Vancomycin-Susceptible S-aureus. Antimicrob. Agents Chemother, 2016; 60: 3730-3742.

2) Iguchi S, Mizutani T, Hiramatsu K, Kikuchi K: Rapid Acquisition of Linezolid Resistance in Methicillin-Resistant Staphylococcus aureus: Role of Hypermutation and Homologous Recombination. PLoS One, 2016; 11: e0155512.

3) Nishizaki N, Nakagawa M, Hara S, Oda H, Kantake M, Obinata K, Uehara Y, Hiramatsu K, Shimizu T: Effect of PMX-DHP for sepsis due to ESBL-producing $\mathrm{E}$. coli in an extremely low-birthweight infant. Pediatr Int, 2016; 58: 411-414.

4) Fudo S, Yamamoto N, Nukaga M, Odagiri T, Tashiro M, Hoshino T: Two Distinctive Binding Modes of Endonuclease Inhibitors to the N-Terminal Region of Influenza Virus Polymerase Acidic Subunit. Biochemistry, 2016; 55: 2646-2660.

5) Suzuki K, Hirai $Y$, Morita F, Uehara $Y$, Oshima H, Mitsuhashi K, Amano A, Naito T: A case of infective endocarditis along with a ruptured valve caused by Streptococcus agalactiae in an immunocompetent man. IDCases, 2016; 6: 94-96.

6) Fukui S, Uehara Y, Fujibayashi K, Takahashi O, Hisaoka T, Naito T: Bacteraemia predictive factors among general medical inpatients: a retrospective cross-sectional survey in a Japanese university hospital. BMJ Open, 2016; 6: e010527.

An asterisk (*) denotes doctoral works by Japanese students.

A dugger $(\dagger)$ denotes doctoral works by non-Japanese students.
7) Nishizaki Y, Yamagami S, Inoue H, Uehara Y, Kobayashi S, Daida H: Reactive Arthritis Caused by Urinary Tract Infection. Intern Med, 2016; 55: 1195-1198.

8) Suzuki A, Uehara Y, Saita M, Inui A, Isonuma H, Naito T: Raltegravir and Abacavir/Lamivudine in Japanese Treatment-Naive and Treatment-Experienced Patients with HIV Infection: a 48-Week Retrospective Pilot Analysis. Jpn J Infect Dis, 2016; 69: 33-38.

9) Yokokawa H, Fukuda H, Suzuki A, Fujibayashi K, Naito T, Uehara Y, Nakayama A, Matsuo H, Sanada H, Jose PA, Miwa Y, Hisaoka T, Isonuma $\mathrm{H}$ : Association Between Serum Uric Acid Levels/Hyperuricemia and Hypertension Among 85,286 Japanese Workers. J Clin Hypertens, 2016; 18: 53-59.

10) Tanaka $Y$, Amano A, Morisaki M, Sato $Y$, Sasaki T: Cellular peptidyl-prolyl cis/trans isomerase Pin1 facilitates replication of feline coronavirus. Antiviral Res, 2016; 126: 1-7.

11) Hishinuma $T$, Katayama $Y$, Matsuo M, Sasaki T, Hiramatsu K: Complete Genome Sequence of Vancomycin-Intermediate Staphylococcus aureus Strain MI (HIP5827). Genome Announc, 2016 17; 4. pii: e00123-16.

12) Morita F, Hirai Y, Suzuki K, Uehara Y, Mitsuhashi K, Amano A, Naito T: Infective endocarditis and Sjögren's syndrome diagnosed simultaneously. IDCases, 2016; 7: 6-8.

13) Hu ZS, Murakami T, Suzuki K, Tamura H, Reich J, Kuwahara-Arai K, Iba T, Nagaoka I: Antimicrobial cathelicidin peptide LL-37 inhibits the pyroptosis of macrophages and improves the survival of polybacterial septic mice. Int Immunol, 2016; 28: 245-253.

14) Hosoda $T$, Uehara $Y$, Matsuda N, Kawase $Y$, Tanei M, Haba Y, Nakamura A, Tabe Y, Naito T, Ohsaka A: Performance evaluation of a

This is a reprint of content originally published in Juntendo University HP. 
novel fully automated real-time reverse transcriptase-polymerase chain reaction kit for the detection of norovirus. Rinsho Byori, 2016; 64: 1347-1352.

\section{Department of Infection Control Science}

〈Orginal Articles〉

1) Nishizaki N, Nakagawa M, Hara S, Oda H, Kantake M, Obinata K, Uehara Y, Hiramatsu K, Shimizu T: Effect of PMX-DHP for sepsis due to ESBL-producing $\mathrm{E}$. coli in an extremely low-birthweight infant. Pediatr Int, 2016; 58: 411-414.

2) Fudo S, Yamamoto N, Nukaga M, Odagiri T, Tashiro M, Hoshino T: Two distinctive binding modes of endonuclease inhibitors to the $\mathrm{N}$-terminal region of influenza virus polymerase acidic subunit. Biochemistry, 2016; 55: 2646-2660.

3) Suzuki K, Hirai Y, Morita F, Uehara Y, Oshima H, Mitsuhashi K, Amano A, Naito T: A case of infective endocarditis along with a ruptured valve caused by Streptococcus agalactiae in an immunocompetent man. IDCases, 2016; 6: 94-96.

4) Fukui S, Uehara Y, Fujibayashi $K$, Takahashi O, Hisaoka T, Naito T: Bacteraemia predictive factors among general medical inpatients: a retrospective cross-sectional survey in a Japanese university hospital. BMJ Open, 2016; 6: e010527.

5) Nishizaki Y, Yamagami S, Inoue H, Uehara Y, Kobayashi S, Daida H: Reactive arthritis caused by urinary tract infection. Intern Med, 2016; 55: 1195-1198.

6) Suzuki A, Uehara Y, Saita M, Inui A, Isonuma $\mathrm{H}$, Naito T: Raltegravir and abacavir/lamivudine in Japanese treatment-naïve and treatment-experienced patients with HIV infection: a 48-week retrospective pilot analysis. Jpn J Infect Dis, 2016; 69: 33-38.

7) Yokokawa H, Fukuda H, Suzuki A, Fujibayashi K, Naito T, Uehara Y, Nakayama A, Matsuo H, Sanada H, Jose PA, Miwa Y, Hisaoka T, Isonuma $\mathrm{H}$ : Association between serum uric acid levels/hyperuricemia and hypertension among 85,286 Japanese workers. J Clin Hypertens, 2016; 18: 53-59.

8) Morita F, Hirai Y, Suzuki K, Uehara Y,
Mitsuhashi K, Amano A, Naito T: Infective endocarditis and Sjögren's syndrome diagnosed simultaneously. IDCases, 2016; 7: 6-8.

9) Hu ZS, Murakami T, Suzuki K, Tamura H, Reich J, Kuwahara-Arai K, Iba T, Nagaoka I: Antimicrobial cathelicidin peptide LL-37 inhibits the pyroptosis of macrophages and improves the survival of polybacterial septic mice. Int Immunol, 2016; 28: 245-253.

10) Hosoda T, Uehara $Y$, Matsuda N, Kawase $Y$, Tanei M, Haba Y, Nakamura A, Tabe Y, Naito T, Ohsaka A: Performance evaluation of a novel fully automated real-time reverse transcriptase-polymerase chain reaction kit for the detection of norovirus. The Official Journal of Japanese Society of Laboratory Medicine, 2016; 64: 1347-1352.

\section{Department of Tropical Medicine and Parasitology}

〈Orginal Articles〉

1) Honma $H$, Niikura $M$, Kobayashi $F$, Horii $T$, Mita T, Endo H, Hirai M: Mutation tendency of mutator Plasmodium berghei with proofreading-deficient DNA polymerase delta. Sci Rep, 2016; 6: 36971.

$\dagger$ 2) Pongvongsa $T$, Nonaka D, Iwagami $M$, Nakatsu M, Phongmany P, Nishimoto F, Kobayashi J, Hongvanthon B, Brey PT, Moji K, Mita T, Kano S: Household clustering of asymptomatic malaria infections in Xepon district, Savannakhet province, Lao PDR. Malar J, 2016; 15: 508.

3) Hashimoto M, Doi M, Kurebayashi N, Furukawa K, Hirawake-Mogi H, Ohmiya Y, Sakurai T, Mita T, Mikoshiba K, Nara T: Inositol 1,4,5-trisphosphate receptor determines intracellular $\mathrm{Ca} 2+$ concentration in trypanosoma cruzi throughout its life cycle. FEBS Open Bio, 2016; 6: 1178-1185.

4) Yatsushiro $S$, Yamamoto $T$, Yamamura $S$, Abe K, Obana E, Nogami T, Hayashi T, Sesei T, Oka H, Okello-Onen J, Odongo-Aginya EI, Alai MA, Olia A, Anywar D, Sakurai M, Palacpac NM, Mita T, Horii T, Baba Y, Kataoka M: Application of a cell microarray chip system for accurate, highly sensitive, and rapid diagnosis for malaria in Uganda. Sci Rep, 2016; 6: 30136.

5) Morales J, Hashimoto M, Williams TA, 
Hirawake-Mogi H, Makiuchi T, Tsubouchi A, Kaga N, Taka H, Fujimura T, Koike M, Mita T, Bringaud F, Concepción JL, Hashimoto T, Embley TM, Nara T: Differential remodelling of peroxisome function underpins the environmental and metabolic adaptability of diplonemids and kinetoplastids. Proc Biol Sci, 2016; 283: 1830.

6) Takamiya S, Mita T: Large-scale purification of active liquid-cultured Caenorhabditis elegans using a modified Baermann apparatus. Parasitol Int, 2016; 65: 580-583.

7) Mita T, Culleton R, Takahashi N, Nakamura M, Tsukahara T, Hunja CW, Win ZZ, Htike WW, Marma AS, Dysoley L, Ndounga M, Dzodzomenyo M, Akhwale WS, Kobayashi J, Uemura H, Kaneko A, Hombhanje F, Ferreira MU, Björkman A, Endo H, Ohashi J: Little polymorphism at the K13 propeller locus in worldwide Plasmodium falciparum populations prior to the introduction of artemisinin combination therapies. Antimicrob Agents Chemother, 2016; 60: 3340-3347.

8) Takamiya S, Hashimoto M, Mita T, Yokota T, Nakajima Y, Yamakura F, Sugio S, Fujimura T, Ueno T, Yamasaki H: Bioinformatic identification of cytochrome b5 homologues from the parasitic nematode Ascaris suum and the free-living nematode Caenorhabditis elegans highlights the crucial role of A. suum adult-specific secretory cytochrome b5 in parasitic adaptation. Parasitol Int, 2016; 65: $113-120$.

9) Hashimoto M, Nara T, Mita T, Mikoshiba K: Morpholino antisense oligo inhibits transsplicing of pre-inositol 1,4,5-trisphosphate receptor mRNA of Trypanosoma cruzi and suppresses parasite growth and infectivity. Parasitol Int, 2016; 65: 175-179.

10) Hoshika $Y$, Takahashi F, Togo S, Hashimoto M, Nara T, Kobayashi T, Nurwidya F, Kataoka H, Kurihara M, Kobayashi E, Ebana H, Kikkawa M, Ando K, Nishino K, Hino O, Takahashi K, Seyama K: Haploinsufficiency of the folliculin gene leads to impaired functions of lung fibroblasts in patients with Birt-Hogg-Dubé syndrome. Physiol Rep, 2016; 4: pii: e13025.

11) Kobayashi I, Takahashi F, Nurwidya F, Nara
T, Hashimoto M, Murakami A, Yagishita S, Tajima K, Hidayat M, Shimada N, Suina K, Yoshioka Y, Sasaki S, Moriyama M, Moriyama $\mathrm{H}$, Takahashi K: Oct4 plays a crucial role in the maintenance of gefitinib-resistant lung cancer stem cells. Biochem Biophys Res Commun, 2016; 473: 125-132.

12) Tsubokawa D, Sugiyama H, Mikami F, Shibata K, Shibahara T, Fukuda K, Takamiya S, Yamasaki H, Nakamura T, Tsuji N: Collection methods of trematode eggs using experimental animal models. Parasitol Int, 2016; 65 (5 Pt B): 584-587.

13) Yamasaki H, Kumazawa H, Sekikawa Y, Oda R, Hongo I, Tsuchida T, Saito N, Morishima Y, Sugiyama H: First confirmed human case of Diphyllobothrium stemmacephalum infection and molecular verification of the synonymy of Diphyllobothrium yonagoense with D. stemmacephalum (Cestoda: Diphyllobothriidea). Parasitol Int, 2016; 65 (5 Pt A) : 412-421.

14) Fujita $T$, Waga $E$, Kitaoka $K$, Imagawa $T$, Komatsu Y, Takanashi K, Anbo F, Anbo T, Katuki S, Ichihara S, Fujimori S, Yamasaki H, Morishima Y, Sugiyama H, Katahira H: Human infection by acanthocephalan parasites belonging to the genus Corynosoma found from small bowel endoscopy. Parasitol Int, 2016; 65: 491-493.

15) Thanchomnang $T$, Tantrawatpan $C$, Intapan PM, Sanpool O, Lulitanond V, Tourtip S, Yamasaki H, Maleewong W: Rapid identification of nine species of diphyllobothriidean tapeworms by pyrosequencing. Sci Rep, 2016; 6: 37228.

16) Janwan P, Intapan PM, Yamasaki H, Rodpai R, Laummaunwai P, Thanchomnang T, Sanpool O, Kobayashi K, Takayama K, Kobayashi Y, Maleewong W: Development and usefullness of an immunochromatographic device to detect antibodies for rapid diagnosis of human gnathostomiasis. Parasit Vectors, 2016; 9: 14.

17) Ebine K, Hirai M, Sakaguchi M, Yahata K, Kaneko O, Saito-Nakano Y: Plasmodium Rab5b is secreted to the cytoplasmic face of the tubovesicular network in infected red blood cells together with $\mathrm{N}$-acylated adenylate kinase 2. Malar J, 2016; 15: 323. 
18) Fougère $A$, Jackson AP, Bechtsi DP, Braks JA, Annoura T, Fonager J, Spaccapelo R, Ramesar J, Chevalley-Maurel S, Klop O, van der Laan AM, Tanke HJ, Kocken CH, Pasini EM, Khan SM, Böhme U, van Ooij C, Otto TD, Janse CJ, Franke-Fayard B: Variant exported blood-stage proteins encoded by Plasmodium multigene families are expressed in liver stages where they are exported into the parasitophorous vacuole. PLoS Pathog, 2016; 12: e1005917.

19) Rijpma SR, van der Velden M, Annoura T, Matz JM, Kenthirapalan S, Kooij TW, Matuschewski K, van Gemert GJ, van de Vegte-Bolmer M, Siebelink-Stoter R, Graumans W, Ramesar J, Klop O, Russel FG, Sauerwein RW, Janse CJ, Franke-Fayard BM, Koenderink JB: Vital and dispensable roles of Plasmodium multidrug resistance transporters during blood- and mosquitostage development. Mol Microbiol, 2016; 101: 78-91.

20) Rijpma SR, van der Velden M, González-Pons M, Annoura T, van Schaijk BC, van Gemert GJ, van den Heuvel JJ, Ramesar J, ChevalleyMaurel S, Ploemen IH, Khan SM, Franetich JF, Mazier D, de Wilt JH, Serrano AE, Russel FG, Janse CJ, Sauerwein RW, Koenderink JB, Franke-Fayard BM: Multidrug ATP-binding cassette transporters are essential for hepatic development of Plasmodium sporozoites. Cell Microbiol, 2016; 18: 369-383.

21) Inaoka DK, Iida $M$, Tabuchi $T$, Honma $T$, Lee N, Hashimoto S, Matsuoka S, Kuranaga T, Sato K, Shiba T, Sakamoto K, Balogun EO, Suzuki S, Nara T, Rocha JR, Montanari CA, Tanaka A, Inoue M, Kita K, Harada S: The open form inducer approach for structurebased drug design. PLoS One, 2016; 11: e0167078.

\section{$\langle$ Reviews〉}

1) Mita $T$, Tachibana $S$, Hashimoto M, Hirai M: Plasmodium falciparum Kelch 13: A potential molecular marker for tackling artemisinin-resistant malaria parasites. Expert Rev Anti Infect Ther, 2016 14: 125-135.

\section{Department of Host Defense and Biochemical Research}

〈Orginal Articles〉

1) Someya A, Ikegami T, Sakamoto K, Nagaoka I: Glucosamine downregulates the IL-1 $\beta-$ Induced expression of proinflammatory cytokine genes in human synovial MH7A cells by $\mathrm{O}-\mathrm{GlcNAc}$ modification-dependent and -independent mechanisms. PLoS One, 2016; 11: e0165158.

* 2) Miyamoto M, Takano M, Aoyama T, Soyama $\mathrm{H}$, Kato M, Yoshikawa T, Shibutani T, Matsuura H, Goto T, Sasa H, Nagaoka I, Furuya K: Is modified radical hysterectomy needed for patients with clinical stage I/II endometrial cancers? A historical control study. Oncology, 2016; 90: 179-185.

3) Inoue S, Nakajima S, Endo M, Nagaoka I: Correlation between lipid peroxide concentration in sserum and intake pattern with a focus on lipid, copper, histidine, and folic acid. J Jpn Mibyo System Association, 2016; 22: $15-28$.

4) Tomonaga A, Fukagawa M, Ikeda H, Hori T, Ohkawara M, Nagaoka I: Evaluation of the effect of the administration of an N-acetylglucosamine-containing green tea supplement on biomarkers for cartilage metabolism in healthy individuals without symptoms of arthritis: a randomized double-blind placebocontrolled clinical study. Functional Foods in Health and Disease, 2016; 6: 788-808.

5) Tomonaga A, Watanabe $K$, Fukagawa M, Suzuki A, Kurokawa M, Nagaoka I: Evaluation of the effect of $\mathrm{N}$-acetyl-glucosamine administration on biomarkers for cartilage metabolism in healthy individuals without symptoms of arthritis: a randomized doubleblind placebo-controlled clinical study. Exp Ther Med, 2016; 12: 1481-1489.

6) Tada H, Shimizu T, Nagaoka I, Takada H: Vitamin D3 analog maxacalcitol (OCT) induces $\mathrm{hCAP}-18 / \mathrm{LL}-37$ production in human oral epithelial cells. Biomed Res, 2016; 37: 199-205.

7) Iba T, Hamakubo T, Nagaoka I, Sato K, Thachil J: Physiological levels of pentraxin 3 and albumin attenuate vascular endothelial 
cell damage Induced by histone $\mathrm{H} 3$ in vitro. Microcirculation, 2016; 23: 240-247.

8) Hu Z, Murakami T, Suzuki K, Tamura H, Reich J, Kuwahara-Arai K, Iba T, Nagaoka I: Antimicrobial cathelicidin peptide LL-37 inhibits the pyroptosis of macrophages and improves the survival of polybacterial septic mice. Int Immunol, 2016; 28: 245-253.

9) Murakami T, Hu Z, Tamura H, Nagaoka I: Release mechanism of high mobility group nucleosome binding protein 1 from lipopolysaccharide-stimulated macrophages. Mol Med Rep, 2016; 13: 3115-3120.

10) Suzuki K, Murakami T, Hu Z, Tamura H, Kuwahara-Arai K, Iba T, Nagaoka I: Human host defense cathelicidin peptide LL-37 enhances the lipopolysaccharide uptake by liver sinusoidal endothelial cells without cell activation. J Immunol, 2016; 196: 1338-1347.

〈Reviews〉

1) Nagaoka I: Have host defense peptides been acting in innate immunity since the trilobites of the Cambrian period 540 million years ago? Juntendo Med J, 2016; 62: 96-97.

2) Hu Z, Nagaoka I: Modulation of macrophage cell death, pyroptosis by host defense peptide LL-37. Juntendo Med J, 2016; 62: 98-104.

3) Suzuki K, Nagaoka I: The effect of human host defense peptide LL-37 on endothelial cells. Juntendo Med J, 2016; 62: 105-111.

4) Tamura H, Reich J, Nagaoka I: Bacterial endotoxin assays relevant to host defense peptides. Juntendo Med J , 2016; 62: 132-140.

\section{Department of Molecular and Cellular Biochemistory}

〈Orginal Articles〉

1) Chiba T, Nakahara T, Hashimoto-Hachiya A, Yokomizo T, Uchi H, Furue M: The leukotriene $\mathrm{B} 4$ receptor $\mathrm{BLT} 2$ protects barrier function via actin polymerization with phosphorylation of myosin phosphatase target subunit 1 in human keratinocytes. Exp Dermatol, 2016; 25: 532-536.

* 2) Ichiki T, Koga T, Okuno T, Saeki K, Yamamoto Y, Yamamoto H, Sakaguchi M, Yokomizo T: Modulation of leukotriene B4 receptor 1 signaling by receptor for advanced glycation end products (RAGE). FASEB J, 2016; 30: 1811-1822.
3) Ishii Y, Saeki K, Liu M, Sasaki F, Koga T, Kitajima K, Meno C, Okuno T, Yokomizo T: Leukotriene B4 receptor type 2 (BLT2) enhances skin barrier function by regulating tight junction proteins. FASEB J, 2016; 30: 933-947.

4) Kojo K, Ito $Y$, Eshima K, Nishizawa N, Ohkubo H, Yokomizo T, Shimizu T, Watanabe M, Majima M: BLT1 signalling protects the liver against acetaminophen hepatotoxicity by preventing excessive accumulation of hepatic neutrophils. Sci Rep, 2016; 6: 29650.

5) Oba J, Nakahara T, Hashimoto-Hachiya A, Liu M, Abe T, Hagihara A, Yokomizo T, Furue M: CD10-Equipped Melanoma Cells Acquire Highly Potent Tumorigenic Activity: A Plausible Explanation of Their Significance for a Poor Prognosis. PLoS One, 2016; 11: e0149285.

* 6) Shigematsu M, Koga T, Ishimori A, Saeki K, Ishii Y, Taketomi Y, Ohba M, Jo-Watanabe A, Okuno T, Harada N, Harayama T, Shindou H, Li J D, Murakami M, Hoka S, Yokomizo T: Leukotriene B4 receptor type 2 protects against pneumolysin-dependent acute lung injury. Sci Rep, 2016; 6: 34560.

7) Tabe Y, Hatanaka Y, Nakashiro M, Sekihara K, Yamamoto S, Matsushita H, Kazuno S, Fujimura T, Ikegami T, Nakanaga K, Matsumoto H, Ueno T, Aoki J, Yokomizo T, Konopleva M, Andreeff M, Miida T, Iwabuchi $\mathrm{K}$, Sasai K: Integrative genomic and proteomic analyses identifies glycerol-3-phosphate acyltransferase as a target of low-dose ionizing radiation in EBV infected-B cells. Int J Radiat Biol, 2016; 92: 24-34.

8) Tatematsu M, Yoshida R, Morioka Y, Ishii N, Funami K, Watanabe A, Saeki K, Seya T, Matsumoto M: Raftlin Controls Lipopolysaccharide-Induced TLR4 Internalization and TICAM-1 Signaling in a Cell Type-Specific Manner. J Immunol, 2016; 196: 3865-3876.

〈Reviews〉

1) Ichiki T, Koga T, Yokomizo T: Receptor for Advanced Glycation End Products Regulates Leukotriene B4 Receptor 1 Signaling. DNA Cell Bio, 2016; 35: 747-750. 


\section{Department of Immunology}

〈Orginal Articles〉

* 1) Haga K, Chiba A, Shibuya T, Osada T, Ishikawa D, Kodani T, Nomura O, Watanabe S, Miyake S: MAIT cells are activated and accumulated in the inflamed mucosa of ulcerative colitis. J Gastroenterol Hepatol, 2016; 31: 965-972.

2) Kadowaki A, Miyake S, Saga R, Chiba A, Mochizuki H, Yamamura T: Gut environment-induced intraepithelial autoreactive CD4 (+) $\mathrm{T}$ cells suppress central nervous system autoimmunity via LAG-3. Nat Commun, 2016; 7: 11639.

* 3) Hayashi E, Chiba A, Tada K, Haga K, Kitagaichi M, Nakajima S, Kusaoi M, Sekiya F, Ogasawara M, Yamaji K, Tamura N, Takasaki Y, Miyake S: Involvement of mucosal-associated invariant $\mathrm{T}$ cells in ankylosing spondylitis. J Rheumatol, 2016; 43: 1695-1703.

4) Finnberg NK, Gokare P, Navaraj A, Lang Kuhs KA, Cerniglia GJ, Yagita H, Takeda K, Motoyama N, El-Deiry WS: Agonists of the TRAIL death receptor DR5 sensitize intestinal stem cells to chemotherapy-induced cell death and trigger gastrointestinal toxicity. Cancer Res, 2016; 76: 700-712.

5) Meng L, Bai Z, He S, Mochizuki K, Purushe J, Sun H, Wang J, Yagita H, Mineishi S, Fung H, Yanik GA, Carcchio R, Fan X, Crisalli LM, Hexner EO, Reshef R, Zhang Y, Zhang Y: The Notch ligand DLL4 defines a capability of human dendritic cells in regulating Th1 and Th17 differentiation. J Immunol, 2016; 196: 1070-1080.

6) Haabeth $\mathrm{OAW}$, Lorvik KB, Yagita H, Bogen $B$, Corthay A: Interleukin-1 is required for cancer eradication mediated by tumorspecific Th1 cells. Oncoimmunology, 2016; 5: e1039763.

7) Chypre M, Seaman J, Cordeiro OG, Willen L, Knoop KA, Buchanan A, Sainson RC, Williams IR, Yagita H, Schneider P, Mueller CG: Characterization and application of two RANK-specific antibodies with different biological activities. Immunol Lett, 2016; 171: 5-14.
8) Baas M, Besançon A, Goncalves T, Valette F, Yagita H, Sawitzki B, Volk HD, WaeckelEnée E, Rocha B, Chatenoud L, You S: TGF $\beta$-dependent expression of $\mathrm{PD}^{-1}$ and PD-L1 controls CD8+ $\mathrm{T}$ cell anergy in transplant tolerance. eLife, 2016; 5: e08133.

9) Cordeiro OG, Chypre M, Brouard N, Rauber S, Alloush F, Romera-Hernandez M, Bénézech C, Li Z, Eckly A, Coles MC, Rot A, Yagita H, Léon C, Ludewig B, Cupedo T, Lanza F, Mueller CG: Integrin-alpha IIb identifies murine lymph node lymphatic endothelial cells responsive to RANKL. PLoS One, 2016; 11: e0151848.

10) Ahrends $T$, Babala N, Xiao Y, Yagita $H$, van Eenennaam H, Borst J: CD27 agonism plus PD-1 blockade recapitulates CD4+ T-cell help in therapeutic anticancer vaccination. Cancer Res, 2016; 76: 2921-2931.

11) Matsuoka S, Ishii $Y$, Nakao A, Abe M, Ohtsuji $\mathrm{N}$, Momose S, Jin H, Arase H, Sugimoto K, Nakauchi Y, Masutani H, Maeda M, Yagita H, Komatsu N, Hino O: Establishment of a therapeutic anti-pan HLA-class II monoclonal antibody that directly induces lymphoma cell death via large pore formation. PLoS One, 2016; 11: e0150496.

12) Starkey MR, Nguyen DH, Brown AC, Essifie AT, Kim RY, Yagita H, Horvat JC, Hansbro PM: Programmed death ligand 1 promotes early-life Chlamydia respiratory infectioninduced severe allergic airway disease. Am J Respir Cell Mol Biol, 2016; 54: 493-503.

13) Smyth MJ, Yagita H, McArthur GA: Combination anti-CTLA-4 and anti-RANKL in metastatic melanoma. J Clin Oncol, 2016; 34: e104-106.

14) Cunningham AL, Guentzel MN, Yu JJ, Hung CY, Forsthuber TG, Navara CS, Yagita H, Williams IR, Klose KE, Eaves-Pyles TD, Arulanandam BP: M-cells contribute to the entry of an oral vaccine but are not essesntial for the subsequent induction of protective immunity against Francisella tularensis. PLoS One, 2016; 11: e0153402.

15) Xue W, Metheringham RL, Brentville VA, Gunn B, Symonds P, Yagita H, Ramage JM, Durrant LG: SCIB2, an antibody DNA vaccine encoding NY-ESO-1 epitopes, 
induces potent antitumor immunity which is further enhanced by checkpoit blockade. Oncoimmunology, 2016; 5: e1169353.

16) Mochizuki K, Meng L, Mochizuki I, Tong Q, He S, Liu Y, Purushe J, Fung H, Zaidi MR, Zhang Y, Reshef R, Blazar BR, Yagita H, Mineishi S, Zhang Y: Programming of donor $\mathrm{T}$ cells using allogeneic delta-like ligand 4-positive dendritic cells to reduce GVHD in mice. Blood, 2016; 127: 3270-3280.

17) Kroon P, Gadiot J, Peeters M, Gasparini A, Deken MA, Yagita H, Verheij M, Borst J, Blank CU, Verbrugge I: Concomitant targeting of programmed death-1 (PD-1) and CD137 improves the efficacy of radiotherapy in a mouse model of human BRAFV600mutant melanoma. Cancer Immunol Immunother, 2016; 65: 753-763.

18) Kondo Y, Ohno T, Nishii N, Harada K, Yagita H, Azuma M: Differential contribution of three immune checkpoint (VISTA, CTLA-4, PD-1) pathways to antitumor responses against squamous cell carcinoma. Oral Oncol, 2016; 57: 54-60.

19) Haw TJ, Starkey MR, Nair PM, Pavlidis S, Liu G, Nguyen DH, Hsu AC, Hanish I, Kim RY, Collison AM, Inman MD, Wark PA, Foster PS, Knight DA, Mattes J, Yagita H, Adcock IM, Horvat JC, Hansbro PM: A pathogenic role for tumor necrosis factor-related apoptosisinducing ligand in chronic obstructive pulmonary disease. Mucosal Immunol, 2016; 9: 859-872.

20) Shimizu K, Yamasaki S, Shinga J, Sato Y, Watanabe T, Ohara O, Kuzushima K, Yagita H, Komuro Y, Asakura M, Fujii S: Systemic DC activation modulates the tumor microenvironment and shapes the long-lived tumorspecific memory mediated by CD8 $+\mathrm{T}$ cells. Cancer Res, 2016; 76: 3756-3766.

21) Kimura Y, Nagai N, Tsunekawa N, SatoMatsushita M, Yoshimoto T, Cua DJ, Iwakura Y, Yagita H, Okada F, Tahara H, Saiki I, Irimura T, Hayakawa Y: IL-17A-producing CD30+ Vס1 T cells drive inflammationinduced cancer progression. Cancer Sci, 2016; 107: 1206-1214.

22) Nair VR, Franco LH, Zacharia VM, Khan HS, Stamm CE, You W, Marciano DK, Yagita H,
Levine B, Shiloh MU: Microfold cells actively translocate Mycobacterium tuberculosis to initiate infection. Cell Rep, 2016; 16: 12531258.

23) Beyranvand Nejad E, van der Sluis TC, van Duikeren S, Yagita H, Janssen GM, van Veelen PA, Melief CJ, van der Burg SH, Arens R: Tumor eradication by cisplatin is sustained by CD80/86-mediated costimulation of CD8 + T cells. Cancer Res, 2016; 76: 6017-6029.

24) Ngiow SF, Young A, Blake SJ, Hill GR, Yagita $\mathrm{H}$, Teng MW, Korman AJ, Smyth MJ: Agonistic CD40 mAb-driven IL-12 reverses resistance to anti-PD1 in a $\mathrm{T}$ cell-rich tumor. Cancer Res, 2016; 76: 6266-6277.

25) Teoh JJ, Gamache AE, Gillespie AL, Stadnisky MD, Yagita H, Bullock TNJ, Brown MG: Acute virus control mediated by licensed NK cells sets primary CD8 $+\mathrm{T}$ cell dependence on CD27 costimulation. J Immunol, 2016; 197: 4360-4370.

26) Xue W, Brentville VA, Symonds P, Cook KW, Yagita H, Metheringham RL, Durrant LG: SCIB1, a huIgG1 antibody DNA vaccination, combined with PD-1 blockade induced efficient therapy of poorly immunogenic tumors. Oncotarget, 2016; 7: 83088-83100.

*27) Kunishige T, Taniguchi H, Terada M, Akiba H, Yagita H, Abe R, Hori J: Protective role of ICOS and ICOS ligand in corneal transplantation and in maintenance of immune privilege. Invest Ophthalmol Vis Sci, 2016; 57: 68156823.

28) Elhai M, Avouac J, Hoffmann-Vold AM, Ruzehaji N, Amiar O, Ruiz B, Brahiti H, Ponsoye M, Fréchet M, Burgevin A, Pezet S, Sadoine J, Guilbert T, Nicco C, Akiba H, Heissmeyer V, Subramaniam A, Resnick R, Molberg Ø, Kahan A, Chiocchia G, Allanore Y: OX40L blockade protects against inflammation-driven fibrosis. Proc Natl Acad Sci U S A, 2016; 113: E3901-3910.

29) Ogawa F, Murphy LC, Malavasi EL, O'Sullivan ST, Torrance HS, Porteous DJ, Millar JK: NDE1 and GSK3B associate with TRAK1 and regulate axonal mitochondrial motility: identification of cyclic AMP as a novel modulator of axonal mitochondrial 
trafficking. ACS Chem Neurosci, 2016; 7: 553-564.

$\langle$ Books $\rangle$

1) Croxford JL, Miyake S: Animal models for the study of neuroimmunological disease. In: Kusunoki S, ed. Neruroimmunological Diseases. Japan: Springer, 2016; 33-54.

\section{Department of Molecular Pathogenesis}

$\langle$ Orginal Articles〉

1) Aizawa $Y$, Shirai $T$, Kobayashi $T$, Hino $O$, Tsujii Y, Inoue H, Kazami M, Tadokoro T, Suzuki T, Kobayashi K, Yamamoto Y: The tuberous sclerosis complex model Eker $(\mathrm{TSC} 2+/-)$ rat exhibits hyperglycemia and hyperketonemia due to decreased glycolysis in the liver. Arch Biochem Biophys, 2016; 590: $48-55$.

2) Aizawa $Y$, Shirai T, Kobayashi T, Hino O, Tsujii Y, Inoue H, Kazami M, Tadokoro T, Suzuki T, Kobayashi K, Yamamoto Y: Metabolic abnormalities induced by mitochondrial dysfunction in skeletal muscle of the renal carcinoma Eker (TSC2+/-) rat model. Biosci Biotechonol Biochem, 2016; 80: 1513-1519.

* 3) Mizutani N, Abe M, Matsuoka S, Kajino K, Wakiya M, Ohtsuji N, Hatano R, Morimoto C, Hino O: Establishment of anti-mesothelioma monoclonal antibodies. BMC Res Notes, 2016; 9: 324

4) Matsuoka S, Ishii $Y$, Nakao A, Abe M, Ohtsuji $\mathrm{N}$, Momose S, Jin H, Arase H, Sugimoto K, Nakauchi Y, Masutani H, Maeda M, Yagita H, Komatsu N, Hino O: Establishiment of a therapeutic anti-Pan HLA-class II monoclonal antibody that directly induces lymphoma cell death via large pore formation. PLoS One, 2016; 11: e0150496.

5) Hoshika Y, Takahashi F, Togo S, Hashimoto M, Nara T, Kobayashi T, Nurwidya F, Kataoka H, Kurihara M, Kobayashi E, Ebana H, Kikkawa M, Ando K, Nishino K, Hino O, Takahashi K, Seyama K: Haploinsufficiency of the folliculin gene leads to impaired functions of lung fibroblasts in patients with Birt-Hogg-Dubé syndrome. Physiol Rep, 2016; 4: e13025.

6) Englund E, Bartoschek M, Reitsma B, Jacobsson L, Escudero-Esparza A, Orimo A,
Leandersson K, Hagerling C, Aspberg A, Storm P, Okroj M, Mulder H, Jirström K, Pietras K, Blom AM: Cartilage oligomeric matrix protein contributes to the development and metastasis of breast cancer. Oncogene, 2016; 35: 5585-55960.

7) Escudero-Esparza A, Bartoschek M, Gialeli C, Okroj M, Owen S, Jirström K, Orimo A, Jiang WG, Pietras K, Blom AM: Complement inhibitor CSMD1 acts as tumor suppressor in human breast cancer. Oncotarget, 2016; 7: 76920-76933.

8) He J, Wang N, Tsurui H, Kato M, Iida M, Kobayashi T: Noninvasive, label-free, threedimensional imaging of melanoma with confocal photothermal microscopy: Differentiate malignant melanoma from benign tumor tissue. Sci Rep, 2016; 6: 30209.

$\langle$ Reviews〉

1) Einama $T$, Kawamata $F$, Kamachi H, Nishihara H, Homma S, Matsuzawa F, Mizukami T, Konishi Y, Tahara M, Kamiyama T, Hino O, Taketomi A, Todo S: Clinical impacts of mesothelin expression in gastrointestinal carcinomas. World J Gastrointest Pathophysiol, 2016; 7: 218-222.

2) Mezawa, Y. and Orimo A: The roles of tumor- and metastasis-promoting carcinomaassociated fibroblasts in human carcinomas. Cell Tissue Res, 2016; 365: 675-89.

\section{Department of Rheumatology}

〈Orginal Articles〉

1) Yamanaka H, Nagaoka S, Lee SK, Bae SC, Kasama T, Kobayashi H, Nishioka Y, Ueki Y, Seto Y, Nishinarita M, Tamura N, Kimura N, Saito K, Tomita T, Nawata Y, Suzuki S, Ishigatsubo Y, Munakata Y, Makino Y, Inoue E, Tanaka Y, Takeuchi T: ENCOURAGE study group: Discontinuation of etanercept after achievement of sustained remission in patients with rheumatoid arthritis who initially had moderate disease activity-results from the ENCOURAGE study, a prospective, international, multicenter randomized study. Mod Rheumatol, 2016; 26: 651-661.

2) Yamada Y, Ogasawara M, Gorai M, Matsuki Y, Murayama G, Sugisaki N, Nemoto T, Ando S, Minowa K, Nakano S, Kon T, Tada K, 
Matsushita M, Yamaji K, Tamura N, Takasaki Y: The synovial grade corresponding to clinically involved joints and a feasible ultrasound-adjusted simple disease activity index for monitoring rheumatoid arthritis. Mod Rheumatol, 2016; 26: 1-6.

3) Hayashi E, Chiba A, Tada K, Haga K, Kitagaichi M, Nakajima S, Kusaoi M, Sekiya F, Ogasawara M, Yamaji K, Tamura N, Takasaki Y, Miyake S: Involvement of Mucosal-associated invariant $\mathrm{T}$ cells in Ankylosing Spondylitis. J Rheumatol, 2016; 43: $1695-1703$.

4) Abe Y, Tamura N, Yang KS, Matsuoka J, Kon T, Yamaji K, Hashimoto H, Tsuda H, Takasaki Y: Predictive factors for morality in elderly Japanese patients with severe microscopic polyangiitis: A retrospective singlecenter study. Mod Rheumatol, Epub 2016 July 11.

5) Kusaoi M, Yamaji K, Ishibe Y, Murayama G, Nemoto T, Sekiya F, Kon T, Ogasawara M, Kempe K, Tamura N, Takasaki Y: Separation of circulating microRNAs using apheresis in patients with systemic lupus erythematosus. Ther Apher Dial, 2016; 20: 348-353.

6) Kitagaichi M, Kusaoi M, Tsukahara T, Murayama G, Nemoto T, Sekiya F, Kon T, Ogasawara M, Kempe K, Yamaji K, Tamura N, Tsuda H, Takasaki Y: Safety and efficacy of the leukocytapheresis procedure in eightyfive patients with rheumatoid arthritis. Transfus Apher Sci, 2016; 55: 225-232.

7) Matsuki-Muramoto Y, Nozawa K, Uomori K, Sekigawa I, Takasaki Y: Bortezomib treatment prevents glomerulosclerosis associated with lupus nephritis in a murine model through suppressive effects on the immune and renin-angiotensin systems. Mod Rheumatol, Epub 2016 May 11.

8) Uomori K, Nozawa K, Ikeda K, Doe K, Yamada Y, Yamaguchi A, Fujishiro M, Kawasaki M, Morimoto S, Takamori K, Sekigawa I, Chan EK, Takasaki Y: A reevaluation of anti-NA-14 antibodies in patients with primary Sjögren's syndrome: Significant role of interferon- $\gamma$ in the production of autoantibodies against NA-14. Autoimmunity, 2016; 49: 345-356.
9) Suzuki S, Nakano S, Ando S, Matsudaira R, Kanai Y, Yamanaka K, Takasaki Y: Hepcidin-25 gives an indication of the therapeutic effectiveness of tocilizumab in rheumatoid arthritis - Relationship between disease activity of rheumatoid arthritis and anemia. Rev Bras Reumatol, Epub 2016 Oct 18.

10) Hachiya $Y$, Kawasaki A, Oka S, Kondo Y, Ito S, Matsumoto I, Kusaoi M, Amano H, Suda A, Setoguchi K, Nagai T, Shimada K, Sugii S, Okamoto A, Chiba N, Suematsu E, Ohno S, Katayama M, Kono H, Hirohata S, Takasaki Y, Hashimoto H, Sumida T, Nagaoka S, Tohma S, Furukawa H, Tsuchiya N: Association of HLA-G 3' Untranslated Region Polymorphisms with Systemic Lupus Erythematosus in a Japanese Population: A CaseControl Association Study. PLoS One, 2016; 11: e0158065.

11) Kawasaki A, Hasebe N, Hidaka M, Hirano F, Sada KE, Kobayashi S, Yamada H, Furukawa H, Yamagata K, Sumida T, Miyasaka N, Tohma S, Ozaki S, Matsuo S, Hashimoto H, Makino H, Arimura Y, Harigai M, Tsuchiya N: Protective Role of HLA-DRB1*13: 02 against Microscopic Polyangiitis and MPOANCA-Positive Vasculitides in a Japanese Population: A Case-Control Study. PLoS One, 2016; 11: e0154393.

12) Nishizaki N, Yamagami S, Inoue H, Uehara $Y$, Kobayashi S, Daida H: Reactive arthritis caused by urinary tract infection: a case report. Internal Med, 2016; 55: 1195-1198.

13) Miyashita $T$, Morimoto S, Fujishiro $M$, Hayakawa K, Suzuki S, Ikeda K, Miyazawa K, Morioka M, Takamori K, Ogawa H, Sekigawa I, Takasaki Y: Inhibition of each module of connective tissue growth factor as a potential therapeutic target for rheumatoid arthritis. Autoimmunity, 2016; 49: 109-114.

14) Yamada $Y$, Nozawa $K$, Nakano S, Mitsuo $Y$, Hiruma K, Doe K, Sekigawa I, Yamanaka K, Takasaki Y: Antibodies to microtubuleassociated protein-2 in the cerebrospinal fluid are a useful diagnostic biomarker for neuropsychiatric systemic lupus erythematosus. Mod Rheumatol, 2016; 26: 562-568.

15) Tsuru T, Tanaka Y, Kishimoto M, Saito K, Yoshizawa S, Takasaki Y, Miyamura T, Niiro 
H, Morimoto S, Yamamoto J, Lledo-Garcia R, Shao J, Tatematsu S, Togo O, Koike T: Safety, pharmacokinetics, and pharmacodynamics of epratuzumab in Japanese patients with moderate-to-severe systemic lupus erythematosus: Results from a phase 1/2 randomized study. Mod Rheumatol, 2016; 26: 87-93.

16) Minowa $\mathrm{K}$, Amano $\mathrm{H}$, Ando S, Watanabe $\mathrm{T}$, Ogasawara M, Kawano S, Kaneko T, Morimoto S, Yamaji K, Tamura N, Tokano Y, Hashimoto H, Takasaki Y: Disease Flare Patterns and Predictors of Systemic Lupus Erythematosus in a Monocentric Cohort of 423 Japanese Patients during a Long-term Follow-up: the JUDE Study. Mod Rheumatol, Epub 2016 Oct 19.

\section{Department of Dermatology and Allergology}

$\langle$ Orginal Articles〉

1) Kamijo M, Wada A, Mineki R, Sakanishi T, Ikeda S: Prostaglandin E receptor 4 inhibition restores UVB-induced downregulation of ATP2A2/SERCA2 in cultured normal human keratinocytes. J Dermatol Sci, 2016; 81: $61-73$.

2) Kamo A, Tominaga M, Matsuda H, Kina K, Kamata Y, Umehara Y, Ogawa H, Takamori K: Neurotropin suppresses itch-related behavior in $\mathrm{NC} / \mathrm{Nga}$ mice with atopic dermatitis-like symptoms. J Dermatol Sci, 2016; 81: 212-215.

3) Teye K, Suga Y, Numata S, Soejima M, Ishii N, Krol RP, Ohata C, Matsuda M, Honma M, Ishida-Yamamoto A, Hamada T, Koda Y, Hashimoto T: A founder deletion of corneodesmosin gene is prevalent in Japanese patients with peeling skin disease: Identification of 2 new cases. J Dermatol Sci, 2016; 82: 134-137.

4) Ise $Y$, Suga $Y$, Okumura K, Negi O, Ishii N, Hashimoto T: Erythematous Variety of Bullous Pemphigoid: Case Report and Literature Review. Acta Derm Venerol, 2016; 96; 3: 412-413.

5) Shimura S, Takai T, Iida H, Maruyama $N$, Ochi H, Kamijo S, Nishioka I, Hara M, Matsuda A, Saito H, Nakae S, Ogawa H, Okumura K, Ikeda S: A case of fingernail onychomycosis due to Aspergillus flavus. Med Mycol J, 2016; 57: E21-25.

* 6) Shimura S, Takai T, Iida H, Maruyama N, Ochi H, Kamijo S, Nishioka I, Hara M, Matsuda A, Saito H, Nakae S, Ogawa H, Okumura K, Ikeda S: Epicutaneous Allergic Sensitization by Cooperation between Allergen Protease Activity and Mechanical Skin Barrier Damage in Mice. Journal of Investigative Dermatology, 2016; 136: 1408-1417.

7) Kanazawa K, Okumura K, Ogawa H, Niyonsaba F: An antimicrobial peptide with angiogenic properties, $\mathrm{AG}-30 / 5 \mathrm{C}$, activates human mast cells through the MAPK and NF- $\mathrm{KB}$ pathways. Immunol Res, 2016; 64: 594-603.

8) Noguchi H, Hiruma M, Maruo K, Jono M, Miyata K, Tanaka H, Tanabe K, Miyazaki Y, Ihn H: Localized cutaneous Cryptococcosis: Summary of reported cases in Japan. Med Mycol J, 2016; 57: E35-39.

9) Kiatsurayanon C, Niyonsaba F, Chieosilapatham P, Okumura K, Ikeda S, Ogawa H: Angiogenic peptide (AG) $-30 / 5 \mathrm{C}$ activates human keratinocytes to produce cytekines/ chemokines and to migrate and proliferative via MrgX receptors. J Dermatol. Sci, 2016; 83: 190-199.

10) Tsunemi Y, Hiruma M: Clinical study of Dermatophyte Test Strip, an immunochromatographic method, to detect tinea unguium dermatophytes. J Dermatol, 2016; 43: 14171423.

11) Kamata $Y$, Kimura U, Matsuda $H$, Tengara $S$, Kamo A, Umehara Y, Iizumi K, Kawasaki H, Suga Y, Ogawa H, Tominaga M, Takamori K: Relationships among plasma granzyme B level, pruritus and dermatitis in patients with atopic dermatitis. J Dermatol Sci, 2016; 84: 266-271.

12) Suzuki M, Hara M, Ichikawa S, Kamijo S, Nakazawa T, Hatanaka H, Akiyama K, Ogawa H, Okumura K, Takai T: Presensitization to Ascaris antigens promotes induction of mite-specific IgE upon mite antigen inhalation in mice. Allergol Int, 2016; 65: 44-51.

13) Nakayama H, Kurihara H, Morita YS, Kinoshita T, Mauri L, Prinetti A, Sonnino S, Yokoyama N, Ogawa H, Takamori K, Iwabuchi K: Lipoarabinomannan binding to 
lactosylceramide in lipid rafts is essential for the phagocytosis of mycobacteria by human neutrophils. Sci Signal, 2016; 9: ra101.

14) Yashiro T, Hara $M$, Ogawa $H$, Okumura $K$, Nishiyama C: Critical role of transcription factor PU.1 in the function of the OX40L/TNFSF4 promoter in dendritic cells. Sci Rep, 2016; 6: 34825.

15) Noguchi A, Tominaga M, Takahashi N, Matsuda H, Kamata Y, Umehara Y, Ko KC, Suga Y, Ogawa H, Takamori K: Differences in therapeutic effects of topically applied corticosteroid and tacrolimus on atopic dermatitis-like symptoms in NC/Nga mice. J Dermatol Sci, Epub 2016 Dec 24.

16) Kamijo S, Suzuki M, Hara M, Shimura S, Ochi H, Maruyama N, Matsuda A, Saito H, Nakae S, Suto H, Ichikawa S, Ikeda S, Ogawa H, Okumura K, Takai T: Subcutaneous allergic sensitization to protease allergen is dependent on mast cells but not IL-33: distinct mechanisms between subcutaneous and intranasal routes. J Immunol, 2016; 196: 3559-3569.

17) Kamijo S, Nunomura S, Ra C, Kanaguchi $Y$, Suzuki Y, Ogawa H, Okumura K, Takai T: Innate basophil IL-4 responses against allergens, endotoxin, and cytokines require the $\mathrm{Fc}$ receptor $\gamma$-chain. J Allergy Clin Immunol, 2016; 137: 1613-1615.

18) Kusube F, Tominaga M, Kawasaki H, Yamakura F, Naito H, Ogawa H, Tomooka Y, Takamori K: Electrophysiological properties of brain-natriuretic peptide- and gastrinreleasing peptide-responsive dorsal horn neurons in spinal itch transmission. Neurosci Lett, 2016; 627: 51-60.

19) Miura R, Kasakura K, Nakano N, Hara M, Maeda K, Okumura K, Ogawa H, Yashiro T, Nishiyama C: Role of PU.1 in MHC class II expression via CIITA transcription in plasmacytoid dendritic cells. PLoS One, 2016; 11: e0154094.

20) Torigoe K, Tominaga M, Ko KC, Takahashi N, Matsuda H, Hayashi R, Ogawa H, Takamori $\mathrm{K}$ : Intrathecal minocycline suppresses itch-related behavior and improves dermatitis in a mouse model of atopic dermatitis. J Invest Dermatol, 2016; 136: 879-881.
21) Honjo A, Nakano N, Yamazaki S, Hara M, Uchida K, Kitaura J, Nishiyama C, Yagita H, Ohtsuka Y, Ogawa H, Okumura K, Shimizu T: Pharmacologic inhibition of Notch signaling suppresses food antigen-induced mucosal mast cell hyperplasia. J Allergy Clin Immunol, Epub 2016 Jul 16.

22) Ko KC, Tominaga M, Kamata Y, Umehara Y, Matsuda H, Takahashi N, Kina K, Ogawa M, Ogawa H, Takamori K: Possible antipruritic mechanism of cyclosporine $\mathrm{A}$ in atopic dermatitis. Acta Derm Venereol, 2016; 96: 624-629.

23) Miyashita T, Morimoto S, Fujishiro M, Hayakawa K, Suzuki S, Ikeda K, Miyazawa K, Morioka M, Takamori K, Ogawa H, Sekigawa I, Takasaki Y: Inhibition of each module of connective tissue growth factor as a potential therapeutic target for rheumatoid arthritis. Autoimmunity, 2016; 49: 109-114.

24) Nakamura $Y$, Nakano N, Ishimaru K, Ando N, Katoh R, Suzuki-Inoue K, Koyanagki S, Ogawa H, Okumura K, Shibata S, Nakao A: Inhibition of IgE-mediated allergic reactions by pharmacologically targeting the circadian clock. J Allergy Clin Immunol, 2016; 137: 1226-1235.

25) Misaki K, Takamura-Enya T, Ogawa H, Takamori K, Yanagida M: Tumour-promoting activity of polycyclic aromatic hydrocarbons and their oxygenated or nitrated derivatives. Mutagenesis, 2016; 31: 205-213.

26) Endo S, Yoshitake H, Tsukamoto H, Matsuura H, Kato K, Sakuraba M, Takamori K, Fujiwara H, Takeda S, Araki Y TEX101, a glycoprotein essential for sperm fertility, is required for stable expression of Ly6k on testicular germ cells. Sci Rep, 2016; 6: 23616.

27) Kumada H, Miyakawa H, Muramatsu T, Ando N, Oh T, Takamori K, Nakamoto H: Efficacy of nalfurafine hydrochloride in patients with chronic liver disease with refractory pruritus: A randomized, doubleblind trial. Hepatol Res, Epub 2016 Nov 24.

28) Kamata $Y$, Tominaga M, Takamori K: Itch in Atopic Dermatitis Management. Curr Probl Dermatol, 2016; 50: 86-93.

29) Uomori K, Nozawa K, Ikeda K, Doe K, Yamada Y, Yamaguchi A, Fujishiro M, 
Kawasaki M, Morimoto S, Takamori K, Sekigawa I, Chan EK, Takasaki Y: A reevaluation of anti-NA-14 antibodies in patients with primary Sjögren's syndrome: Significant role of interferon $-\gamma$ in the production of autoantibodies against NA-14. Autoimmunity, 2016; 49: 347-356.

30) Yoshitake H, Oda R, Yanagida M, Kawasaki Y, Sakuraba M, Takamori K, Hasegawa A, Fujiwara H, Araki Y: Identification of an anti-sperm auto-monoclonal antibody (Ts4) -recognized molecule in the mouse sperm acrosomal region and its inhibitory effect on fertilization in vitro. J Reprod Immunol, 2016; 115: 6-13.

31) Fukaura R, Takeichi T, Okuno Y, Kojima D, Kono M, Sugiura K, Suga Y, Akiyama M: Striate Palmoplantar Keratoderma Showing Transgrediens in a Patient Harbouring Heterozygous Nonsense Mutations in both DSG1 and SERPINB7. ACta Derm Venereol, Epub 2016 Oct 27.

〈Reviews〉

1) Takagi A, Kamijo M, Ikeda S: Darier Disease. J Dermatol, 2016; 43: 275-279.

\section{Department of General Medicine}

$\langle$ Orginal Articles〉

* 1) Haba Y, Naito T, Isonuma H: Analysis of 1,044 Cases of Eosinophilia at the Department of General Medicine. 日本病院総合診療 医学会雑誌, 2016; 10: 1-9.

2) Takahashi H, Kutasy B, Friedmacher F, Takahashi T, Puri P: Expression of hepatic lipid droplets is decreased in the nitrofen model of congenital diaphragmatic hernia. Pediatr Surg Int, 2016; 32: 155-160.

3) Fujibayashi K, Hayashi M, Yokokawa H, Naito T: Changes in antidiabetic prescription patterns and indicators of diabetic control among 200,000 patients over 13 years at a single institution in Japan. Diabetol Metab Syndr, 2016; 8: 72.

* 4) Kudo N, Yokokawa H, Fukuda H, Hisaoka T, Isonuma H, Naito T: Analysis of Associations between health literacy and healthy lifestyle characteristics among Japanese outpatients with lifestyle-related disorders. J Gen Famil Med, 2016; 17: 299-306.
5) Okamoto A, Yokokawa H, Sanada H, Naito T: Changes in levels of biomarkers associated with adipocyte function and insulin and glucagon kinetics during treatment with dapagliflozin among obese type 2 diabetes mellitus (T2DM) patients. Drugs R D, 2016; 16: $255-261$.

6) Fujibayashi K, Gunji T, Yokokawa H, Naito T, Sasabe N, Okumura M, Iijima K, Shibuya K, Hisaoka T, Fukuda H: The Relationships between Metabolic Disorders (Hypertension, Dyslipidemia, and Impaired Glucose Tolerance) and Computed Tomography-Based Indices of Hepatic Steatosis or Visceral Fat Accumulation in Middle-Aged Japanese Men. PLoS One, 2016; 11: e0149689.

7) Yokokawa H, Yuasa M, Nedsuwan S, Moolphate S, Fukuda H, Kitajima T, Minematsu K, Tanimura S, Marui E: Daily salt intake estimated by overnight urine collections and basic characteristics of participants with a high cardiovascular disease risk in Thailand Daily salt intake estimated by overnight urine collections indicates a high cardiovascular disease risk in Thailand. Asia Pac J Clin Nutr, 2016; 25: 39-45.

8) Yokokawa H, Fukuda H, Yuasa M, Sanada H, Hisaoka T, Naito T: Association between health literacy and metabolic syndrome or healthy lifestyle characteristics among community-dwelling Japanese people. Diabetol Metab syndr, 2016; 8: 30.

9) Fujibayashi K, Hayashi M, Yokokawa H, Naito T: Changes in antidiabetic prescription patterns and indicators of diabetic control among 200,000 patients over 13 years at a single institution in Japan. Diabetol Metab Syndr, 2016; 8: 72.

10) Yokokawa H, Yuasa M, Nedsuwan S, Moolphate S, Fukuda H, Kitajima T, Minematsu K, Tanimura S, Marui E: Daily salt intake estimated by overnight urine collections indicates a high cardiovascular disease risk in Thailand. Asia Pac J Clin Nutr, 2016; 25: 39-45.

11) Naito T: Should inflammatory markers be used in the diagnosis of a fever of unknown origin? Intern Med, 2016; 55: 1407.

12) Yokokawa H, Fukuda H, Suzuki A, Fujibayashi K, Naito T, Uehara Y, Nakayama A, 
Matsuo H, Sanada H, Jose PA, Miwa Y, Hisaoka T, Isonuma H: Association between serum uric acid levels/hyperuricemia and hypertension among 85,286 Japanese workers. J Clin Hypertens (Greenwich), 2016; 18: 53-59.

13) Suzuki A, Uehara $Y$, Saita M, Inui A, Isonuma H, Naito T: Raltegravir and abacavir/lamivudine in Japanese treatment-naïve and treatment-experienced patients with HIV infection: a $48-$ week retrospective pilot analysis. Jpn J Infect Dis, 2016; 69: 33-38.

14) Inui $A$, Naito $T$ : A case of IgG4-related disease admitted with CRP elevation of unknown origin. Juntendo Medical Journal, 2016; 62: 228-231.

15) Naito T: Clinical approach to febrile patients. Juntendo Medical Journal, 2016; 62: 224-227.

16) Morita F, Hirai Y, Suzuki K, Uehara Y, Mitsuhashi K, Amano A, Naito T: Infective endocarditis and Sjögren's syndrome diagnosed simultaneously. IDCases, 2016; 22: 6-8.

17) Liao CW, Fu CJ, Kao CY, Lee YL, Chen PC, Chuang TW, Naito T, Chou CM, Huang YC, Bonfim I, Fan CK: Prevalence of intestinal parasitic infections among school children in capital areas of the Democratic Republic of São Tomé and Príncipe, West Africa. Afr Health Sci, 2016; 16: 690-697.

18) Suzuki K, Hirai Y, Morita F, Uehara Y, Oshima H, Mitsuhashi K, Amano A, Naito T: A case of infective endocarditis along with a ruptured valve caused by Streptococcus agalactiae in an immunocompetent man. IDCases, 2016; 6: 94-96.

19) Fukui $S$, Uehara $Y$, Fujibayashi K, Takahashi O, Hisaoka T, Naito T: Bacteraemia predictive factors among general medical inpatients: a retrospective cross-sectional survey in a Japanese university hospital. BMJ Open, 2016; 6: e010527.

20) Okamoto A, Yokokawa H, Sanada H, Naito T: Changes in levels of biomarkers associated with adipocyte function and insulin and glucagon kinetics during treatment with dapagliflozin among obese Type 2 diabetes mellitus patients. Drugs R D, 2016; 16: 255261.

21) Kawasaki A, Hasebe N, Hidaka M, Hirano F,
Sada KE, Kobayashi S, Yamada H, Furukawa H, Yamagata K, Sumida T, Miyasaka N, Tohma S, Ozaki S, Matsuo S, Hashimoto H, Makino H, Arimura Y, Harigai M, Tsuchiya N: Protective Role of HLA-DRB1*13: 02 against Microscopic Polyangiitis and MPO-ANCAPositive Vasculitides in a Japanese Population: A Case-Control Study. PLoS One, 2016; 11: e0154393.

22) Nishizaki N, Yamagami S, Inoue H, Uehara Y, Kobayashi S, Daida H: Reactive arthritis caused by urinary tract infection: a case report. Intern Med, 2016; 55: 1195-1198.

\section{Department of Clinical Pharmacology}

$\langle$ Orginal Articles〉

1) Kada A, Yonemoto N, Yokoyama H, Nonogi H; J-PULSE III Investigators, Hanada H, Hase M, Sakamoto T, Kasaoka S, Kikuti M, Nagao K, Sase K, Kimura K, Sumiyoshi T, Fujimoto K, Hisao O, Shirai S, Kanemitsu M, Hayashi K; J-PULSE III Investigators: Association between accessibility to emergency cardiovascular centers and cardiovascular mortality in Japan. Int J Qual Health Care, 2016; 28: 281-287.

〈Reviews〉

1) IMDRF Patient Registries Working Group: Principles of International System of Registries Linked to Other Data Sources and Tools, Final Document, International Medical Device Regulators Forum (IMDRF). International Medical Device Regulators Forum (IMDRF), 30 September 2016. http://www . imdrf.org/docs/imdrf/final/technical/imdrftech-160930-principles-system-registries.pdf

\section{Department of Sports Medicine}

$\langle$ Orginal Articles〉

* 1) Maruyama-Nagao A, Sakuraba K, Suzuki Y: Seasonal variations in vitamin $\mathrm{D}$ status in inand outdoor Japanese female athletes. Biomed Rep, 2016; 5: 113-117.

2) Suzuki Y, Fukushima M, Sakuraba K, Sawaki K, Sekigawa K: Krill oil improves mild knee joint pain: A randomized control trial. PLoS One, 2016; 11: e0162769.

* 3) Endo S, Sakuraba K, Kubota A: Effect of local cooling and blood flow restriction on muscle 
weakness and atrophy caused by detraining. Juntendo Medical Journal, 2016; 62: 146-152.

\section{Department of Epidemiology and Environmental Health}

〈Orginal Articles〉

1) Hosokawa M, Sugaya C, Tsunoda M, Kodama Y, Sugita-Konishi Y, Ohta H, Yokoyama K: The effects of fluoride on the bones and teeth from ICR-derived glomerulonephritis (ICGN) mice and ICR mice after subacute exposure. Fluoride, 2016; 49: 417-428.

2) Matsumoto M, Todo H, Akiyama T, HirataKoizumi M, Sugibayashi K, Ikarashi Y, Ono A, Hirose A, Yokoyama K: Risk assessment of skin lightening cosmetics containing hydroquinone. Regul Toxicol Pharmacol, 2016; 81: 128-135.

3) Takeshige H, Ueno Y, Sasaki F, Namera A, Matsukawa T, Yokoyama K, Hattori N: Acute hippocampal and chronic diffuse white matter involvement in severe methanol intoxication. Neurology, 2016; 87: 2382-2383.

4) Takehara A, Aoyama $Y$, Kurosawa M, Shirafuji Y, Umemura H, Kamiya K, Ushigome Y, Kano Y, Shiohara T, Iwatsuki K: Longitudinal analysis of antibody profiles against plakins in severe drug eruptions: emphasis on correlation with tissue damage in drug-induced hypersensitivity syndrome and drug reaction with eosinophilia and systemic symptoms. Br J Dermatol, 2016; 175: 944-952.

5) Komatsu S, Itoh H, Urakawa K, Yokoyama K: Determinants of sense of coherence in male Japanese workers: a cross-sectional study. Juntendo Medical Journal, 2016; 62: 318-322.
6) Vigeh M, Nishioka E, Yokoyama K, Ohtani K, Matsukawa T: Increased prenatal blood manganese may induce gestational blood pressure. Hypertens Pregnancy, 2016; 35: 583-592.

7) Takahashi S, Katada J, Daida H, Kitamura F, Yokoyama K: Effects of mineralocorticoid receptor antagonists in patients with hypertension and diabetes mellitus: a systematic review and meta-analysis. J Hum Hypertens, 2016; 30: 534-542.

8) Yamamoto K, Yokoyama K, Matsukawa T, Kato S, Kato S, Yamada K, Hirota T: Efficacy of prolonged ingestion of Lactobacillus acidophilus L-92 in adult patients with atopic dermatitis. J Dairy Sci, 2016; 99: 5039-5046.

9) Dewi N, Mi P, Yanagie H, Sakurai $Y$, Morishita Y, Yanagawa M, Nakagawa T, Shinohara A, Matsukawa T, Yokoyama K, Cabral H, Suzuki M, Sakurai Y, Tanaka H, Ono K, Nishiyama N, Kataoka K, Takahashi $\mathrm{H}$ : In vivo evaluation of neutron capture therapy effectivity using calcium phosphate based nanoparticles as Gd DTPA delivery agent. J Cancer Res Clin Oncol, 2016; 142: 767-775.

10) Kitazawa T, Yokoyama K, Kubota K: Combination therapy of glucagon-like peptide-1 receptor agonists and insulin for patients who developed diabetes after partial pancreatectomy. J Diabetes Investig, 2016; 7: 381-385.

11) Kimura E, Matsuyoshi C, Miyazaki $W$, Benner S, Hosokawa M, Yokoyama K, Kakeyama M, Tohyama C: Prenatal exposure to bisphenol A impacts neuronal morphology in the hippocampal CA1 region in developing and aged mice. Arch Toxicol, 2016; 90: 691-700. 